Research Paper:

\title{
Prevalence of Menstrual Disorders and its Relationship With Quality of Life in Female High School Students
}

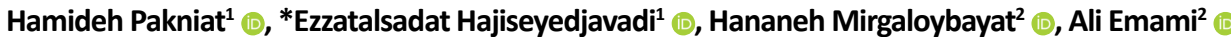

1. Department of Obstetrics and Gynecology, Clinical Research Development Unit, Kosar Hospital, Qazvin University of Medical Sciences, Qazvin, Iran. 2. Student Research Committee, Qazvin University of Medical Sciences, Qazvin, Iran.

\begin{tabular}{|c|c|}
\hline $\begin{array}{l}\text { Use eourd device to scan } \\
\text { and read the article online }\end{array}$ & Citation Pakniat H, Hajiseyedjavadi E, Mirgaloybayat H, Emami A. Prevalence of Menstrual Disorders and its Relationship With \\
\hline aiting & $\begin{array}{l}\text { Quality of Life in Female High School Students. Journal of Inflammatory Diseases. 2020; 24(4):346-355. https://doi.org/10.32598/ } \\
\text { JQUMS.24.4.4 }\end{array}$ \\
\hline 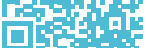 & doi https://doi.org/10.32598/JQUMS.24.4.4 \\
\hline
\end{tabular}

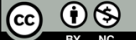

Received: 14 Jun 2020

Accepted: 23 Sep 2020 Available Online: 01 Oct 2020

Keywords:

Menstrual disorders, Quality of Life, High school students

\section{A B STRACT}

Background Menstrual disorders in female students in the early years after menarche affect their physical, emotional and social functions.

Objective This study aimed to investigate the prevalence of menstrual disorders among female high school students and its relationship with their Quality of Life.

Methods This cross-sectional study was conducted on 1000 high school girls in Qazvin, Iran during 2017 2018. Their menstrual disorders and quality of life were evaluated by a demographic checklist and KIDSCREEN questionnaire, respectively. Data were analyzed using independent t-test.

Findings The mean age of high school girls was $15.9 \pm 0.93$ years. The most common disorder was Premenstrual Syndrome (PMS) with a prevalence of $60 \%$. Their quality of life had a significant relationship with PMS, menorrhagia, metorrhagia, dysmenorrhea, spotting, hypermenorrhea $(P<0.001)$, polymenorrhea $(P=0.005)$, hypomnorrhea $(P=0.002)$ and amenorrhea $(P=0.025)$. The quality of life of girls with these disorders were poorer compared to the girls with no menstrual disorder.

Conclusion Menstrual disorders are highly prevalent among female students, and have a considerable impact on their quality of life. Since this impact has the potential to have longer-term consequences, more efforts are needed to address and treat menstrual disorders in female students.

\section{Extended Abstract}

\section{Introduction}

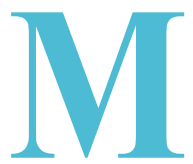

enstruation is a monthly physiological process. Menstrual disorders may occur under the influence of various factors [1]. Studies have shown that the twothirds of women have regular menstrual cycles [2]. Menstrual disorders are one of the main complaints of women which, in addition to having negative effect on their performance, affects their family and society
[3]. Primary dysmenorrhea is defined as cramping pain in the lower abdomen occurring just before or during menstruation, in the absence of other diseases. Its prevalence in Iran was estimated at 85\% [4]. Premenstrual Syndrome (PMS) refers to emotional and physical symptoms that regularly occur in the one to two weeks before the start of each menstrual period. Symptoms resolve within a few days of the onset of bleeding [5].

Primary Amenorrhea is defined as the failure of menses by age 15-16 years or the absence of secondary sexual characteristics by age 14 years [8]. The term oligomenor-

\section{* Corresponding Author:}

Ezzatalsadat Hajiseyedjavadi

Address: Department of Obstetrics and Gynecology, Clinical Research Development Unit, Kosar Hospital, Qazvin University of Medical Sciences, Qazvin, Iran. Tel: +98 (912) 1822806

E-Mail: dr_seidjavadi@yahoo.com 
rhea is used for irregular menstrual periods, and polymenorrhea is a term used to describe menstrual cycles with intervals shorter than 21 days [11]. Metrorrhagia is uterine bleeding at irregular intervals, particularly between the expected menstrual periods, and menorrhagia refers to the blood loss of $80 \mathrm{~mL}$ per a menstrual cycle [12]. Other terms are hypomenorrhea (bleeding for 2-3 days) and hypermenorrhea (bleeding for longer than 7 days) [2].

\section{Materials and Methods}

This cross-sectional study was carried out on 1000 high school girls during 2017-18. They were selected from 20 high schools in Qazvin, Iran by a stratified random sampling technique (using random number table). Inclusion criteria were: Being female, and studying in high school. The girls with chronic systemic diseases and those with no willingness to participate in the study were excluded from the study. Data were collected by a demographic checklist and the KIDSCREEN questionnaire for measuring the subjects' quality of life. Cronbach's alpha coefficient for different dimensions of this questionnaire has been reported 0.78-0.84 [16]. The collected data were analyzed in SPSS V. 16 software using descriptive statistics, chi-square test, and independent t-test by considering a significance level at 0.05 .

\section{Results}

The participants had a Mean $\pm \mathrm{SD}$ age of $15.9 \pm 0.93$ years and a Mean \pm SD body mass index of $21.76 \pm 3.27 \mathrm{Kg} / \mathrm{m}^{2}$.

Table 1. The relationship between having various menstrual disorders and quality of life in high school students

\begin{tabular}{|c|c|c|c|c|}
\hline Menstrual Disorder & Quality of Life & No. & Scores & Sig. \\
\hline \multirow{3}{*}{ Premenstrual Syndrome* } & No menstrual irregularities & 156 & $60.01 \pm 12.66$ & \multirow{3}{*}{$<0.001$} \\
\hline & & & & \\
\hline & With PMS & 600 & $54.84 \pm 11.96$ & \\
\hline \multirow{3}{*}{ Menorrhagia * } & No menstrual irregularities & 156 & $60.01 \pm 12.66$ & \multirow{3}{*}{$<0.001$} \\
\hline & & & & \\
\hline & With menorrhagia & 383 & $55.01 \pm 12.23$ & \\
\hline \multirow{3}{*}{ Metrology* } & No menstrual irregularities & 156 & $60.01 \pm 12.66$ & \multirow{3}{*}{$<0.001$} \\
\hline & & & & \\
\hline & Suffering from metrorrhagia & 356 & $55.19 \pm 11.77$ & \\
\hline \multirow{3}{*}{ Dysmenorrhea * } & No menstrual irregularities & 156 & $60.01 \pm 12.66$ & \multirow{3}{*}{$<0.001$} \\
\hline & & & & \\
\hline & With dysmenorrhea & 266 & $54.42 \pm 12.30$ & \\
\hline \multirow{3}{*}{ Spotting * } & No menstrual irregularities & 156 & $60.01 \pm 12.66$ & \multirow{3}{*}{$<0.001$} \\
\hline & & & & \\
\hline & Suffering from spotting & 224 & $54.29 \pm 11.74$ & \\
\hline \multirow{3}{*}{ Hypermenorrhea * } & No menstrual irregularities & 156 & $60.01 \pm 12.66$ & \multirow{3}{*}{$<0.001$} \\
\hline & & & & \\
\hline & With hypermenorrhea & 190 & $54.27 \pm 12.65$ & \\
\hline \multirow{3}{*}{ Hypomenorrhea * } & No menstrual irregularities & 156 & $60.01 \pm 12.66$ & \multirow{3}{*}{0.002} \\
\hline & & & & \\
\hline & With hypomenorrhea & 24 & $51.31 \pm 12.06$ & \\
\hline \multirow{3}{*}{ Polymenorrhea * } & No menstrual irregularities & 156 & $60.01 \pm 12.66$ & \multirow{3}{*}{0.005} \\
\hline & & & & \\
\hline & Suffering from polymenorrhea & 76 & $54.97 \pm 12.81$ & \\
\hline \multirow{3}{*}{ Amenorrhea * } & No menstrual irregularities & 156 & $60.01 \pm 12.66$ & \multirow{3}{*}{0.025} \\
\hline & & & & \\
\hline & With amenorrhea & 16 & $52.37 \pm 14.9$ & \\
\hline \multirow{3}{*}{ Oligomenorrhea } & No menstrual irregularities & 156 & $60.01 \pm 12.66$ & \multirow{3}{*}{0.074} \\
\hline & & & & \\
\hline & With amenorrhea & 32 & $55.61 \pm 12.27$ & \\
\hline
\end{tabular}


Their Mean \pm SD age at menarche was $12.94 \pm 1.39$ years. Moreover, $84.4 \%$ of them had menstrual disorders, where the PMS was the most common disorder (60\%). Table 1 shows the results of examining relationship between menstrual disorders and the quality of life in girls. The quality of life score of those with menstrual disorders was significantly lower compared to those with no any menstrual disorder, but there was no statistically significant relationship between the quality of life and oligomenorrhea $(\mathrm{P}=0.074)$.

\section{Conclusion}

This purpose of this study was to investigate the prevalence of menstrual disorders and its effect on the quality of life of high school girls $(n=1000)$. The most common disorder was reported PMS with a prevalence of $60 \%$. In Azurah et al.'s study, the most common menstrual disorder was dysmenorrhea (34.8\%) [17]. This discrepancy may be due to difference in the study population; in our study, it consisted of all female students in high schools, while in Azurah et al.'s study, it consisted of females referred to a gynecology center for treatment of menstrual disorders. Many patients with a menstrual disorder such as PMS may not seek treatment. Difference in the number of samples and their economic status can also be effective.

The quality of life of female students in our study had a statistically significant relationship with having PMS, menorrhagia, metrorrhagia, dysmenorrhea, spotting, hypermenorrhea, polymenorrhea, hypomenorrhea and amenorrhea. The quality of life of girls with these disorders was significantly poorer than that of those with no any menstrual disorder. A study conducted by Shahin et al. on 1008 students in Turkey also showed that the quality of life score of girls with PMS were significantly lower than girls who had no any menstrual disorder [19]. In our study, the results revealed that menstrual disorders had a significant negative impact on the quality of life of female students.

Due to the high prevalence of menstrual disorders among female high school students, and the association of these disorders in the early years after menarche with some serious gynecological diseases, menstrual disorders in adolescent girls need to be diagnosed and treated. Screening and treatment of these disorders can prevent their long-term complications. Furthermore, due to the significant negative impact of menstrual disorders on the quality of life of high school girls, it is recommended to provide psychological counseling to them to reduce the psychological burden of these disorders. Further studies are recommended to investigate the causes and eliminate them. It is also suggested that more studies be conducted on the effectiveness of treating menstrual disorders in improving the quality of life in adolescent girls.

\section{Ethical Considerations}

\section{Compliance with ethical guidelines}

This study is approved by the Research Ethics Committee of Qazvin University of Medical Sciences (Code: IR.QUMS.REC.1395.262).

\section{Funding}

This study was extracted from the MD. thesis of third author approved by Qazvin University of Medical Sciences.

\section{Authors' contributions}

Selecting the subject of study and summarizing the contents: Hamideh Pakniat and Ezzatalsadat Hajiseyedjavadi, Writing and analyzing data: Hananeh Mirgaloybayat and Ali Emami, Editing, supervising and project management: Ezzatalsadat Hajiseyedjavadi, Draft Article: Ali Emami.

\section{Conflicts of interest}

The authors declare no conflict of interest.

\section{Acknowledgements}

The authors would like to thank the Clinical Research Development Center of Kosar Hospital in Qazvin and the Student Research Committee of Qazvin University of Medical Sciences. 


\section{شيوع اختلالات قاعدكى و ارتباط آن با كيفيت زندتى دانش آموزان دبيرستانى شهر قزوين}

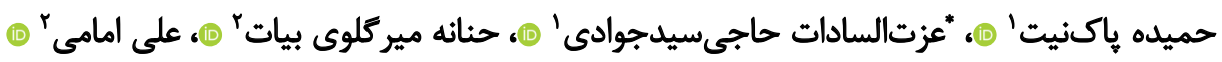

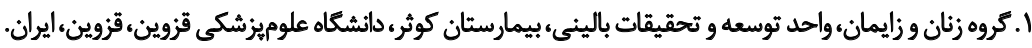

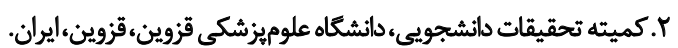

آثان تأثير بسزايى فارد.

هدف مطالعه حاضر با هدف بررسى شيوع اختلالات قاعدكى در بين دانش آموزان دبيرستانى شهر قزوين و ارتباط آن باكيفيت زندكى

صورث مكرفت.

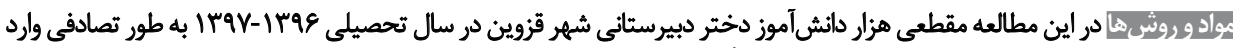

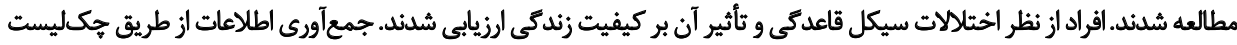

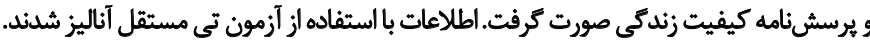

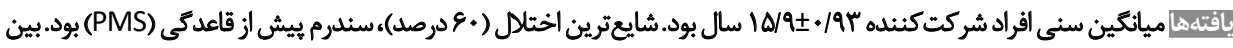

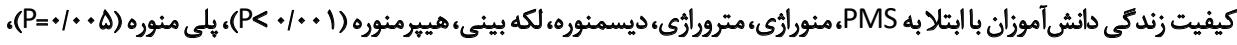

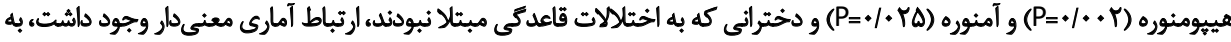

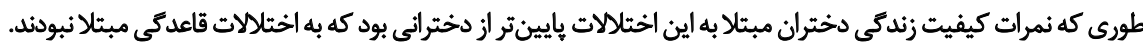

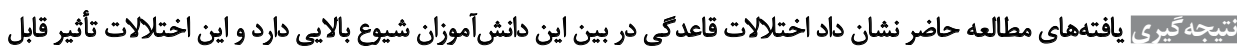

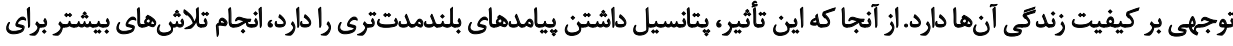

شيوع اين اختلال در ايران تا هم درصد كزارش شده است [F].

مalod

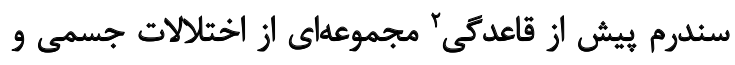

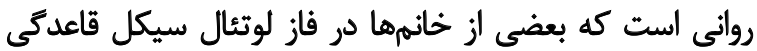

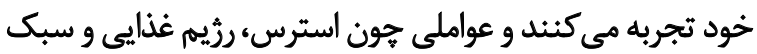

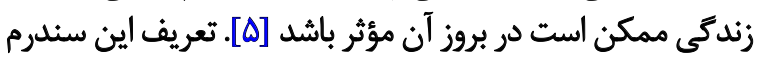

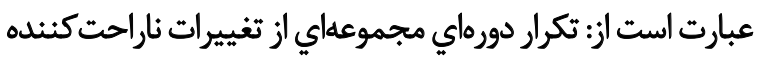

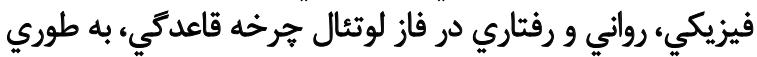

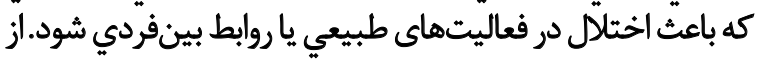

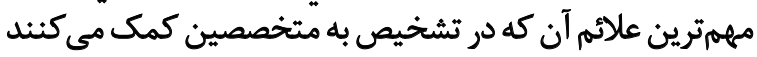

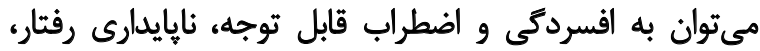

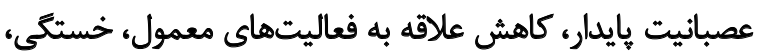

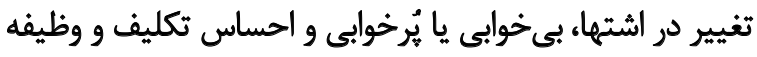

2. Premenstrual Syndrome (PMS)
قاعدكى يك روند فيزيولوزيك ماهانه است و تحت تأثير عوامل

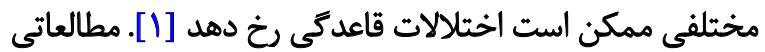

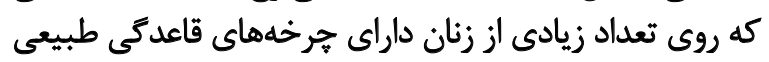

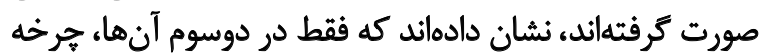

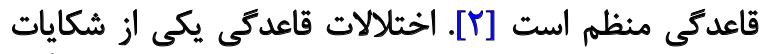

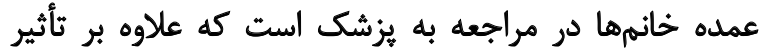

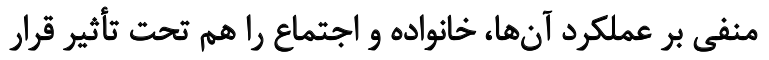

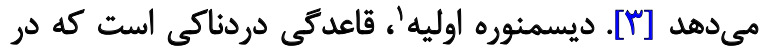

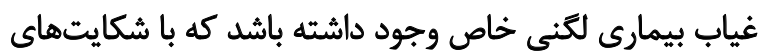

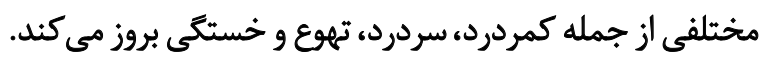
اين اختلال دليل اصلى غيبت از مدرسه درد بين نوجوانان است.

\section{Primary dysmenorrhea}

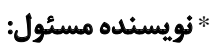

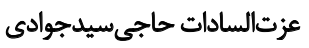

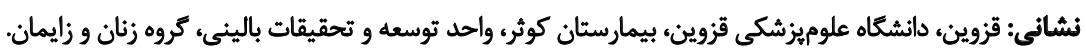

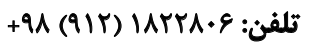
رايانامه: dr_seidjavadi@yahoo.com 


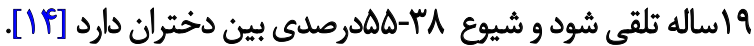

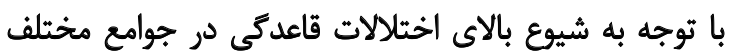

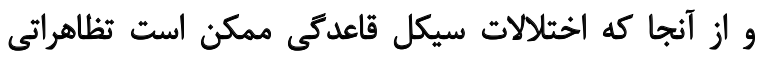

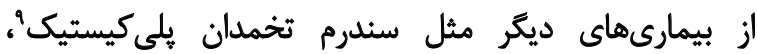

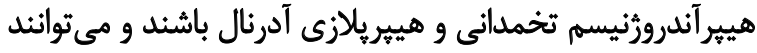

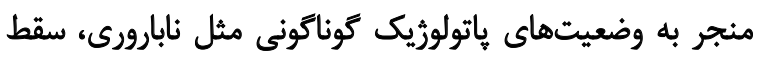

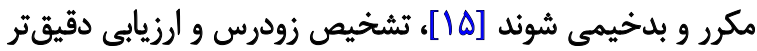

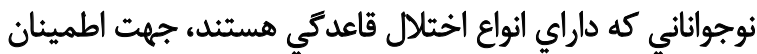
از سلامت آنان ضروري به نظر مىرسد.

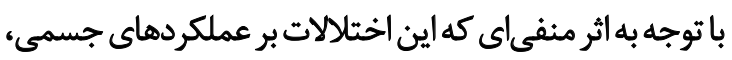

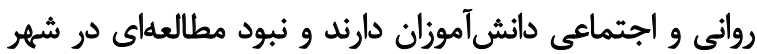

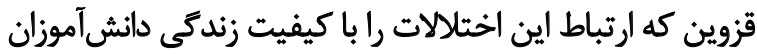

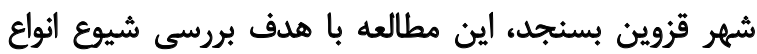

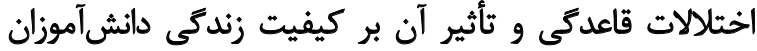
دبيرستانى شهر قزوين انجام شد أندات

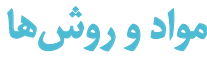

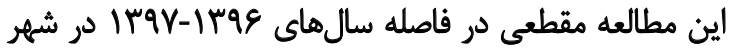

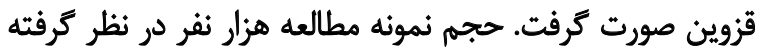

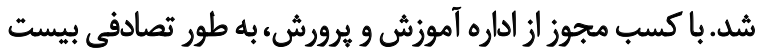

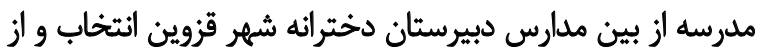

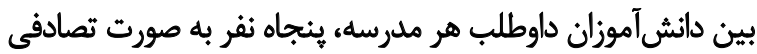

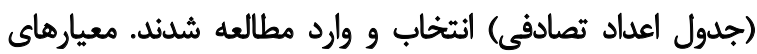

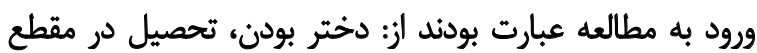

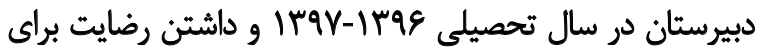

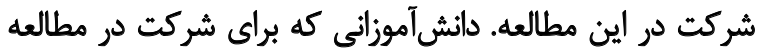

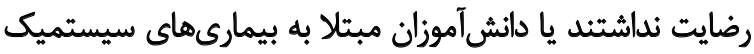

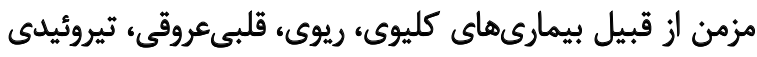

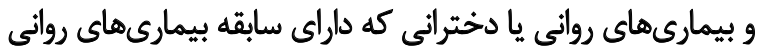

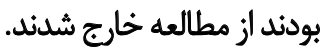

جك ليستى براى مشخصات جمعيتشناختى و سابقه اختلالات

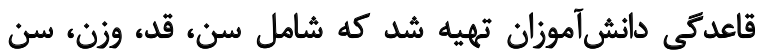

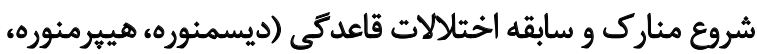

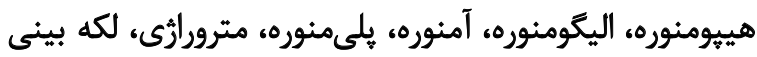

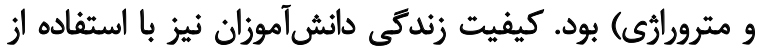

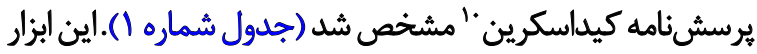

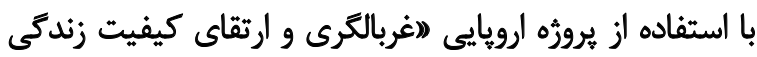

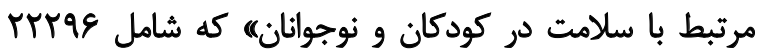

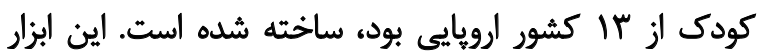

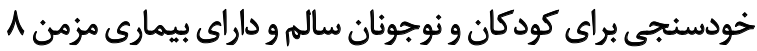

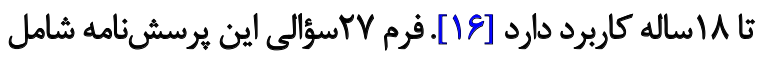

بيش از حد يا خارج از كنترل فرد اشاره كرد [ع]. شيوع اين

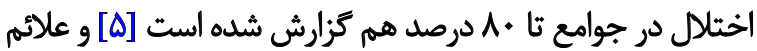

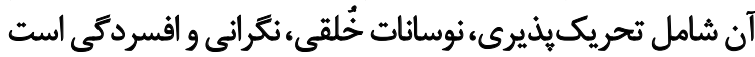

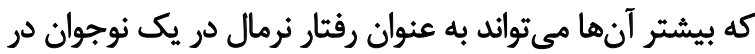

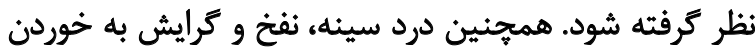

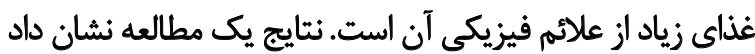

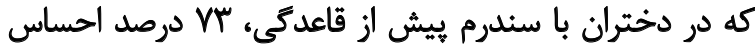

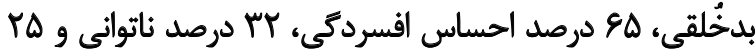

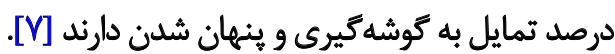

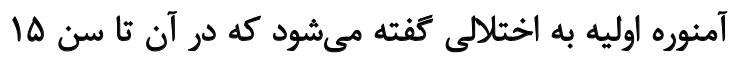

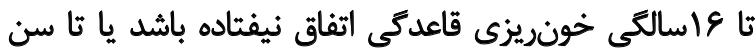

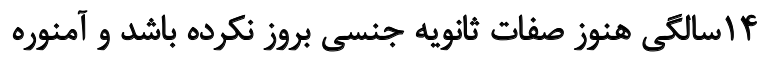

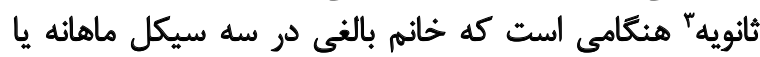

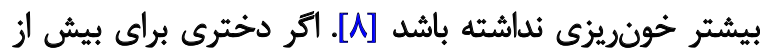

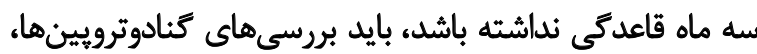

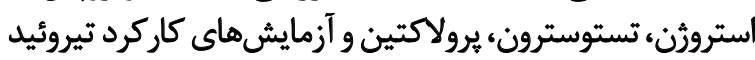

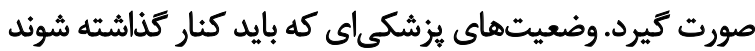

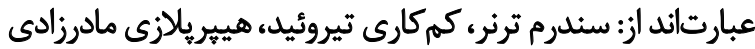

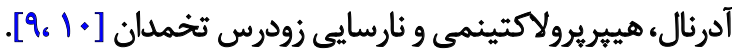

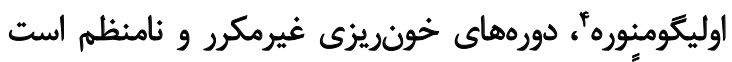

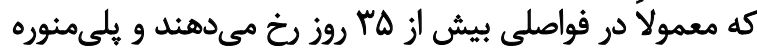

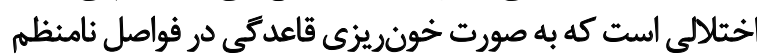

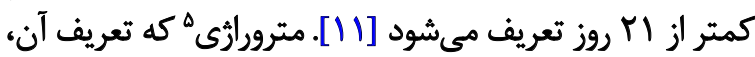

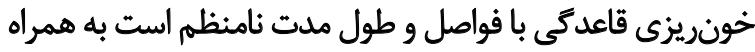

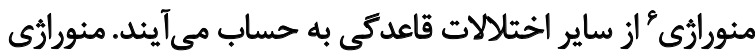

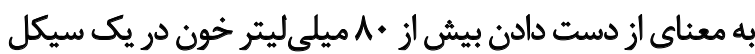

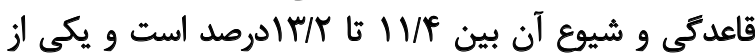

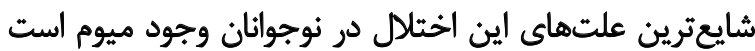

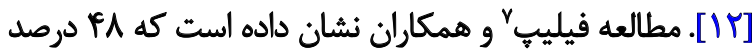

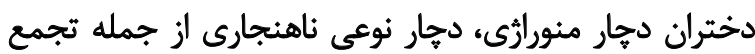

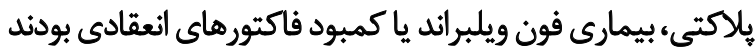

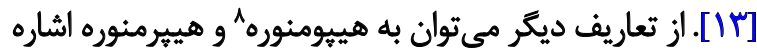

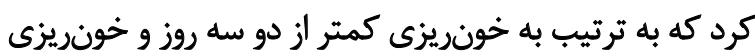

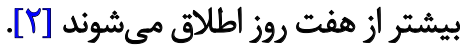

نقص در محور هييوتالاموس هييوفيز تخمدان، شايعترين توضيح

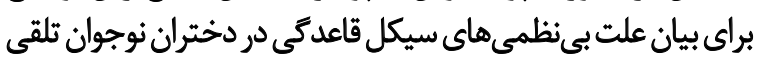

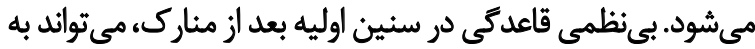

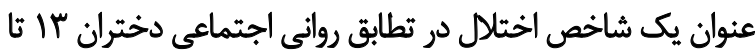

\footnotetext{
3. Secondary amenorrhea

4. Oligomenorrhea

5. Metrorrhagia

6. Menorrhagia

7. Philipp

8. Hypomenorrhea
} 
بيشترين درصد فراوانى ( (9 درصد) را داشت (شكل شماره ()).

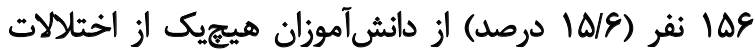

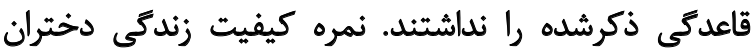

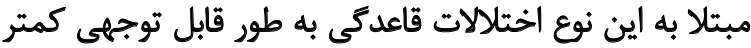

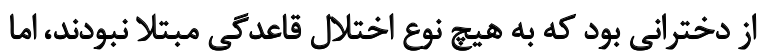

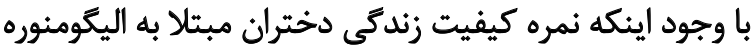

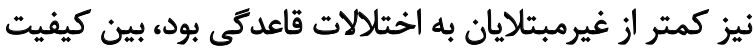

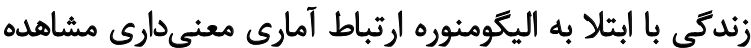
نشد (جدول شماره (Y) (P= (P)

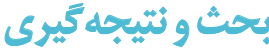

در مطالعه حاضر شيوع اختلالات قاعدكى و تأثير آن بر دير كيفيت زندگى در هزار دانشآموز دبيرستانى با ميانكين سنى

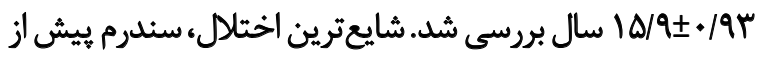

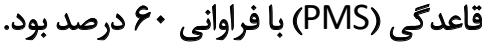

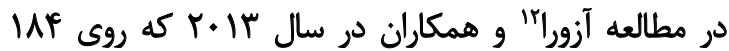

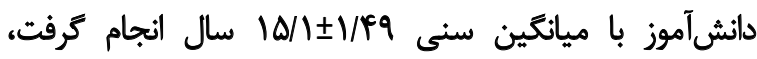

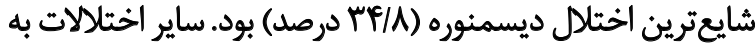

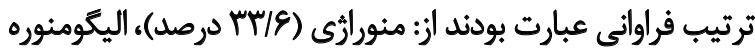

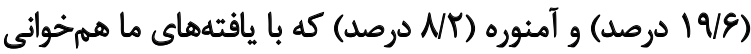

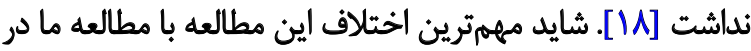

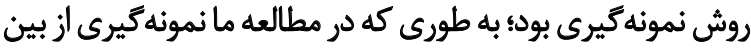

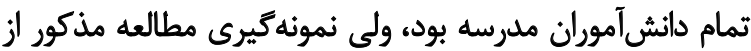

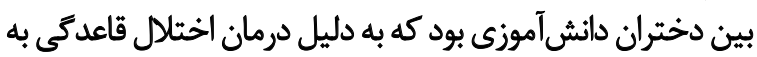

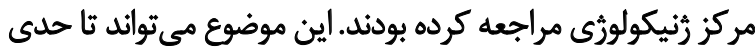

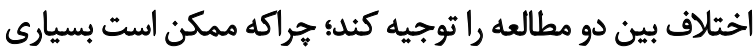

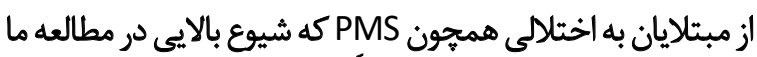

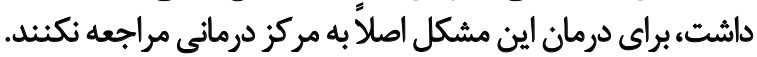

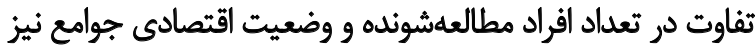
مى توانند اختلاف بين دو مطالعه را توجيه كنند.
ينج بُعد است كه عبارتاند از: ا. بهزيستى جسمى (ينج سؤال):

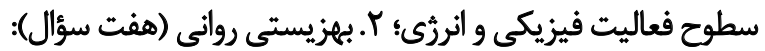

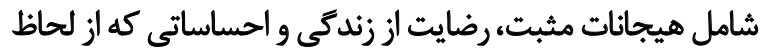

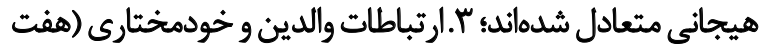

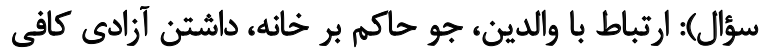

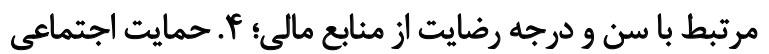

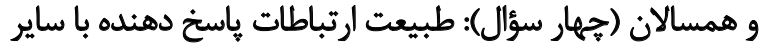

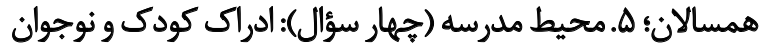

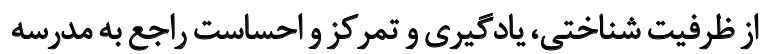

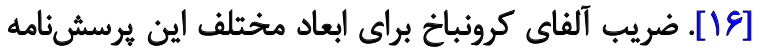

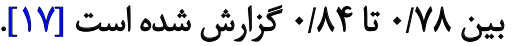
بعد از جلسه آموزشى درباره انواع اختلالات قاعدكى و تعاريف

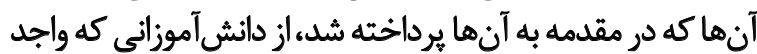

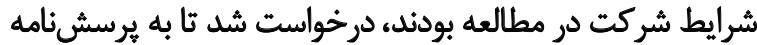

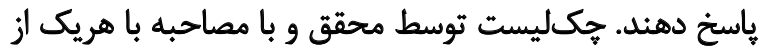

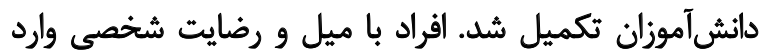

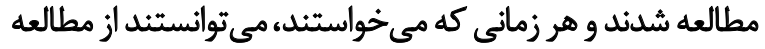

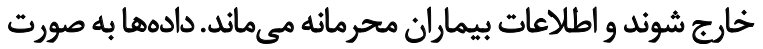

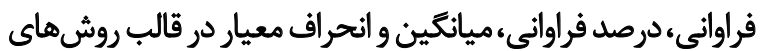

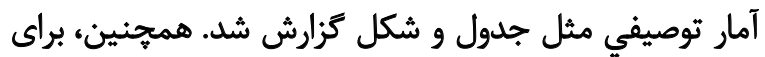

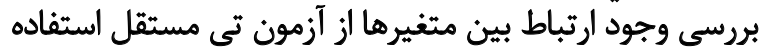

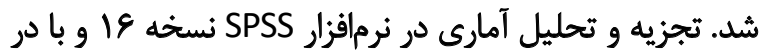
نظر ترفتن سطح معنيداري هـ • انجام شد.

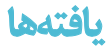

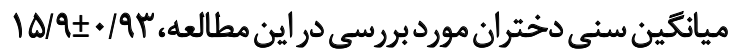

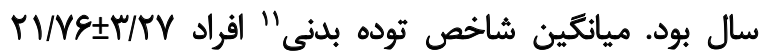

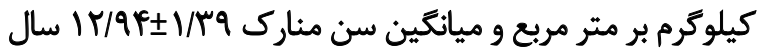

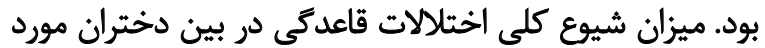

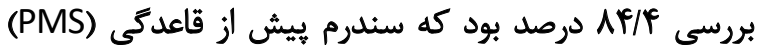

12. Azurah

11. Body Mass Index (BMI)

جدول (. ميانكين نمرات داثش آموزان مورد بروسي (هزار نفر) در برسشينامه كيفيت زندكى مرتبط با سلامت (كيداسكرين) نمره

مقياس

\begin{tabular}{|c|c|}
\hline نمره & مقياس \\
\hline PMYTEYNAI & بهزيستى جسمى \\
\hline$\Delta V / .9 \pm T N N$ & بهزيستى روانى \\
\hline ET/TVETY/NA & ارتباط با والدين و خودمختارى \\
\hline SI/TA+TE/19 & حمايت اجتماعى و همسالان \\
\hline Qr/ArETY/Ve & محيط ملرسه \\
\hline$\Delta \Delta / R q \pm I T / r$ & كيفيت زندكى كل \\
\hline
\end{tabular}

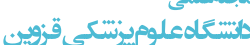


جدول r. ارتباط بين ابتلا به انواع اختلالات قاعدىى با كيفيت زندكى در دانش آموزان دبيرستانى

\begin{tabular}{|c|c|c|c|c|}
\hline سطح معنى دارى & 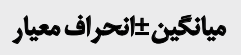 & تعداد & كيفيت زندمى & اختلال قاعدىى \\
\hline \multirow{2}{*}{$<+/ * 1$} & $9.1 .1 \pm 14 / 8$ & 108 & بلون اختلال قاعدىى & \multirow{2}{*}{ ساز قاعدمى" } \\
\hline & $\Delta F / A F \pm I V / Q$ & $9 .$. & مبتلا به PMS & \\
\hline \multirow{2}{*}{$<\cdot / \cdot \cdot 1$} & $8.1+1 \pm 1 r / 98$ & 109 & بدون اختلالل قاعدكى & \multirow{2}{*}{ منوراثىى" } \\
\hline & $\Delta \Delta / \cdot| \pm| r / r r$ & ז" & مبتلا به منورازىى & \\
\hline \multirow{2}{*}{$<+1 .+1$} & $9.1 .1 \pm 1 \% / 98$ & 108 & بدون اختطالل قاعدكى & \multirow{2}{*}{ متروراثى" } \\
\hline & $\Delta \Delta / 19 \pm 1 \backslash / W$ & $r \Delta$ & مبتلا به متروراثى & \\
\hline \multirow{2}{*}{$<+1 . .1$} & $9.1 \cdot 1 \pm 1 \% / 98$ & 108 & بدون اختلال قاعدىى & \multirow{2}{*}{ ديسمنوره" } \\
\hline & $\Delta F / F Y \pm I Y / T$. & reg & مبتلا به ديسمنوره & \\
\hline \multirow{2}{*}{$<\cdot / .+1$} & $9 . / .1 \pm 1 \% / 98$ & 108 & بدون اختلال قاعدىى & \multirow{2}{*}{ لكه بينى" } \\
\hline & $\Delta f / r q \pm \| 1 / M e$ & $m$ & مبثلا به لكه بينى & \\
\hline \multirow{2}{*}{$<+1 . .1$} & $9.1 .1 \pm 14 / 89$ & 100 & بلدون اختالال قاعدكى & \multirow{2}{*}{ هييرمنورة" } \\
\hline & $\Delta F / T Y \pm I T / G \Delta$ & 19. & مبثلا به هييرمنوره & \\
\hline \multirow{2}{*}{$\% r$} & $9.1+1 \pm 1 \% / 98$ & 109 & بدون اختلال قاعدىى & \multirow{2}{*}{ هييومنوره" } \\
\hline & $\Delta V / T M \pm \mid Y / . \varepsilon$ & $\eta$ & مبثلا به هييومتوره & \\
\hline \multirow{2}{*}{$\% \Delta$} & $8 . / .1 \pm \mid r / 98$ & 105 & بدون اختلالل قاعدمىى & \multirow{2}{*}{ يلمهنوره" } \\
\hline & $\Delta \leftarrow / a Y \pm|r / A|$ & ve & مبثلا به بلىمنووره & \\
\hline \multirow{2}{*}{$\% . r \Delta$} & $9.1 \cdot 1 \pm \mid r / 98$ & 108 & بدون اختلال قاعدىى & \multirow{2}{*}{ أمنوره" } \\
\hline & $\Delta r / T r \pm 1 Y / q$ & 18 & مبتلا به آمثوره & \\
\hline \multirow[t]{2}{*}{$\% \cdot r \varphi$} & $9 . / .1 \pm 1 r / 98$ & 108 & بدون اختلال قاعدىى & \multirow{2}{*}{ اليكومنوره } \\
\hline & $\Delta D / 91 \pm 1 Y / T V$ & $\pi$ & مبئلا به أمثوره & \\
\hline
\end{tabular}

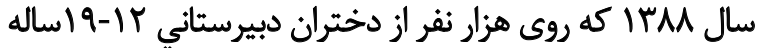

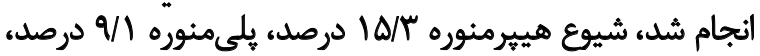

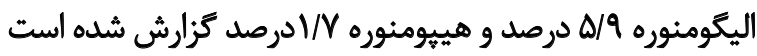

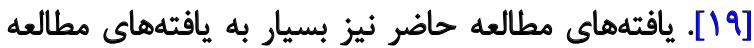

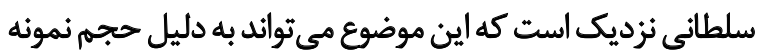
يكسان در دو مطالعه باشد.

بررسىها در خصوص ارتباط بين ابتلا به انواع اختلالات

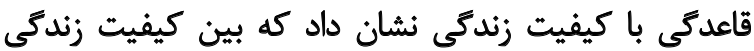

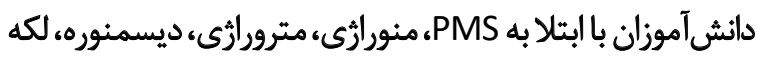

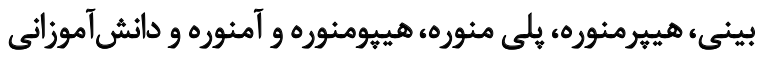

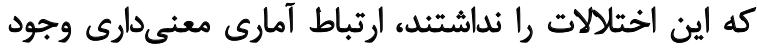

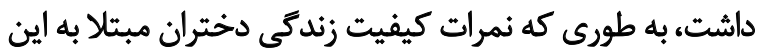

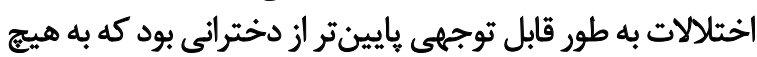

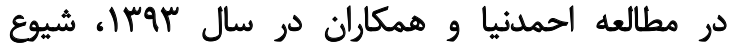

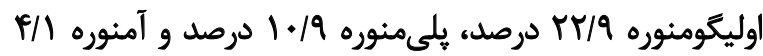

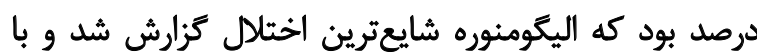

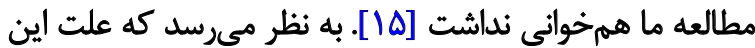

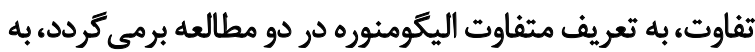

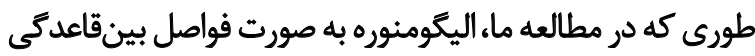

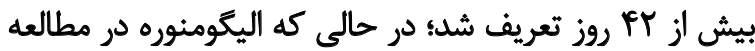

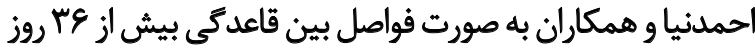

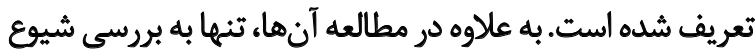

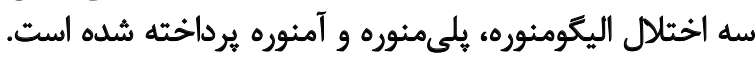

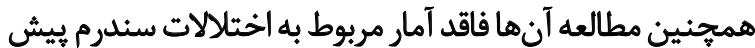

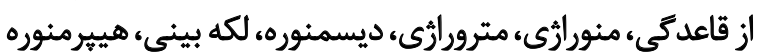

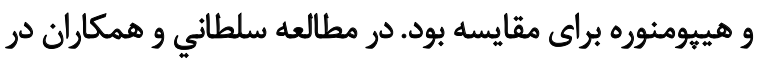




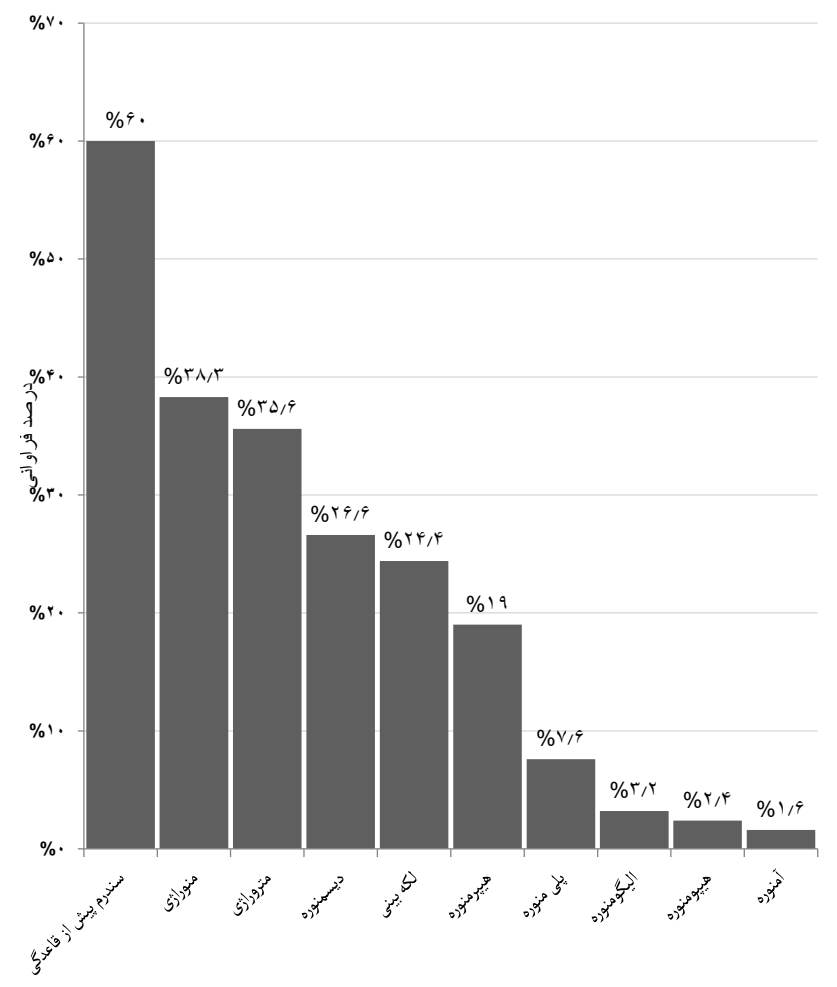

همكارى

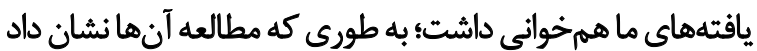

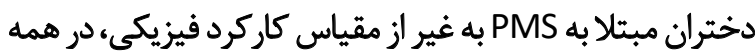

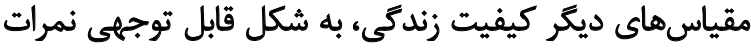
بايينترى نسبت به دختران سالم دارند [Trك].

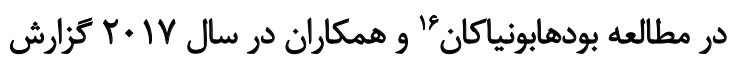

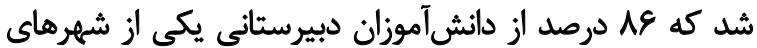

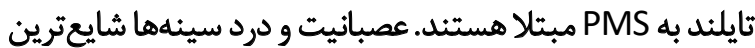

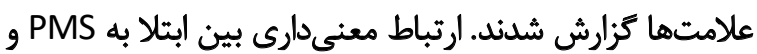

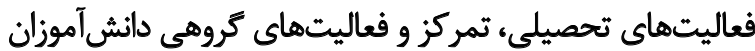

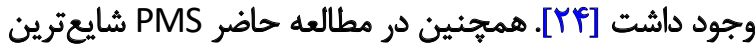

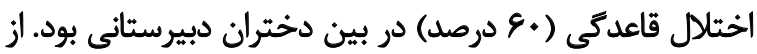

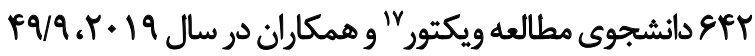

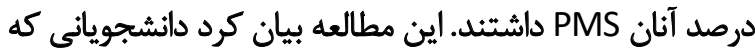

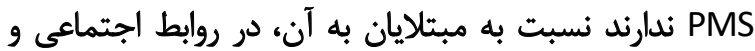

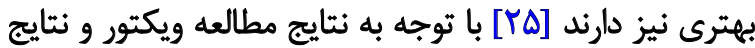

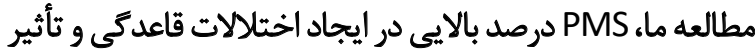

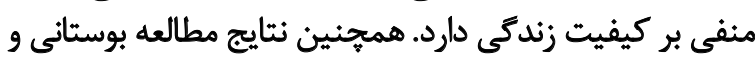

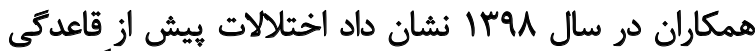
در دانشآموزان دبيرستانى همانند مطالعه حاضر نسبتاً بالاست

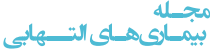

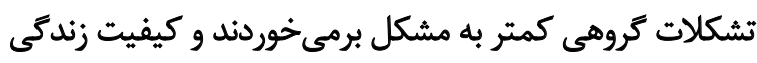

شكل ا. فراوائى انواع اختلالات قاعدكى در بين دختران مورد بررسى

نوع اختلال قاعدكى مبتلانبودند. مطالعهاى كه توسط شاهين

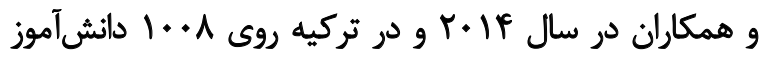

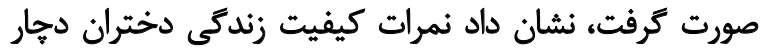

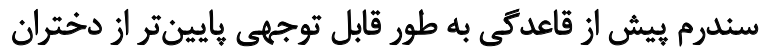

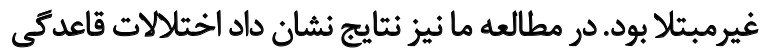

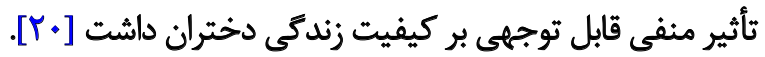

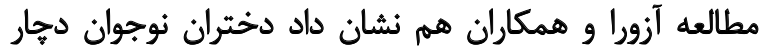

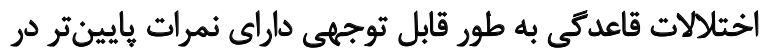

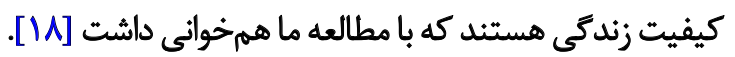

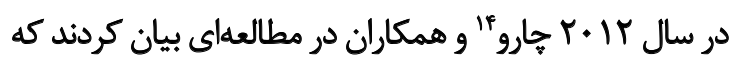

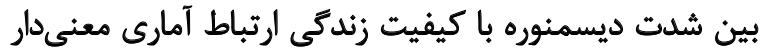

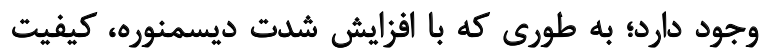

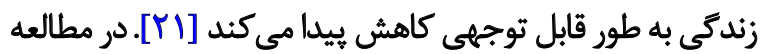

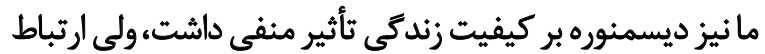

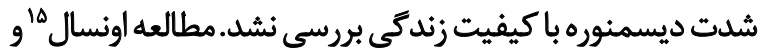

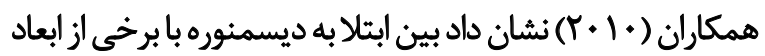

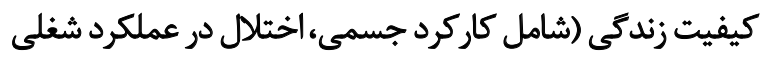

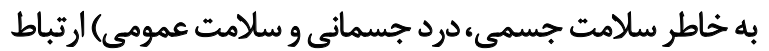

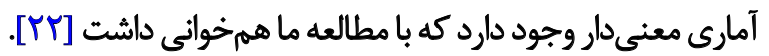
يافتههاى مطالعه دلآرا و همكاران در سال r. r كه با
15. Unsal 


\section{مشاركت نويسندكًان}

انتخاب موضوع مطالعه و جمعبندى مطالب: حميده بإى نيت

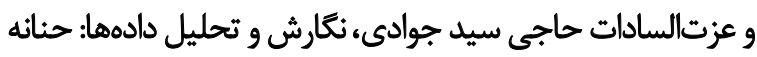

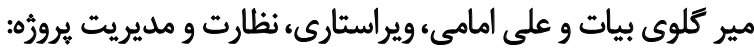

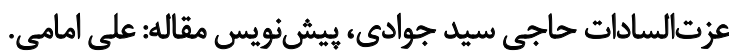

$$
\text { تعارض مئافع }
$$

بنابر اظهار نويسندكان اين مطالعه تعارض منافع ندارد.

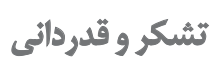

بدينوسيله از همكارى واحد توسعه تحقيقات بالينى بيمارستان

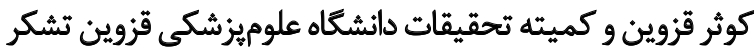

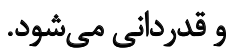

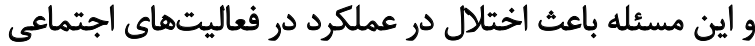

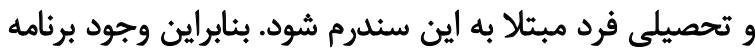

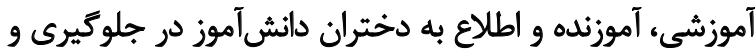

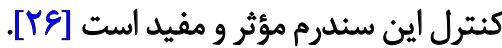

با توجه به شيوع بالاي اختلالات قاعدكى در بين دانشآموزان آمنان

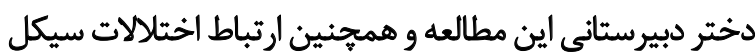

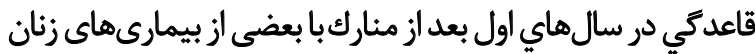

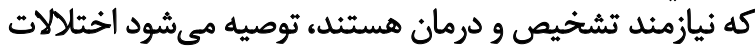

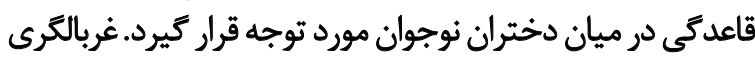

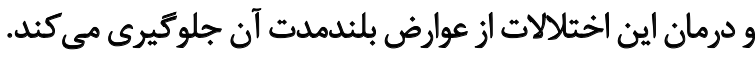

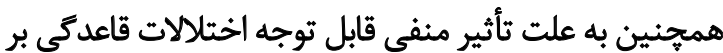

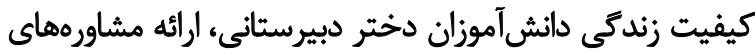

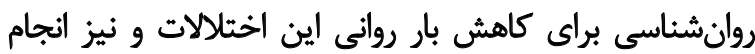

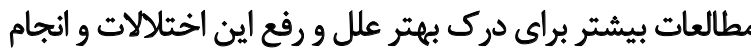

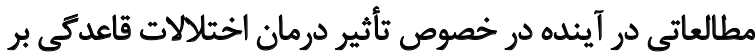

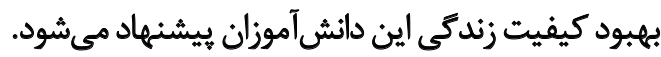

نقاط قوت اين مطالعه جامعه آمارى بالاى آن و بررسى همد

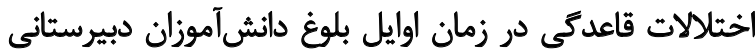

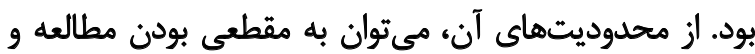

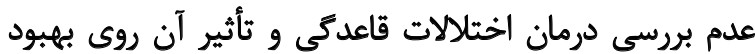

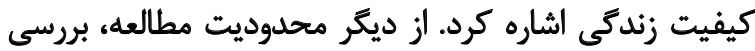

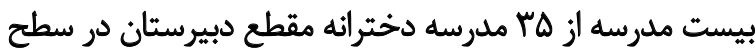

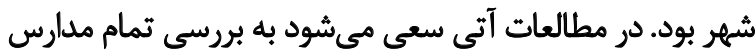

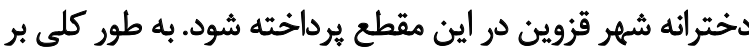

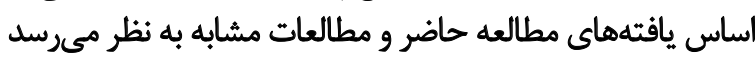

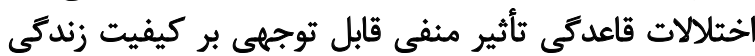

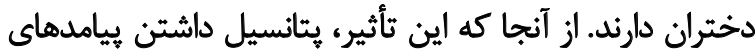

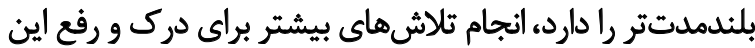

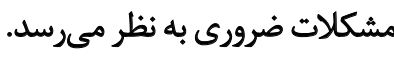

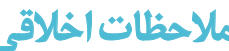

\section{بيروى أز أصول اخلاق يثوهش}

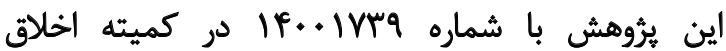

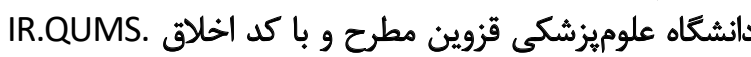

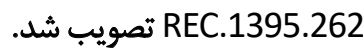

$$
\text { حامي مالي }
$$

اين مقاله بركرفته از بايايانامه عمومى يزشكى حنى حنانه ميركلوى

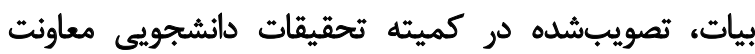

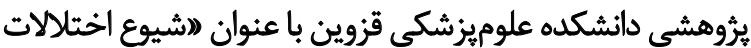

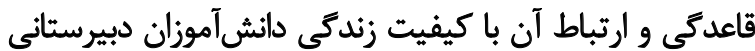
شهر قزوينه است. 


\section{References}

[1] Shiferaw MT, Wubshet M, Tegabu D. Menstrual problems and associated factors among students of Bahir Dar University, Amhara National Regional State, Ethiopia: A cross-sectional survey. Pan Afr Med J. 2014; 17:246. [DOI:10.11604/ pamj.2014.17.246.2230] [PMID] [PMCID]

[2] Marshburn PB, Hurst BS, editors. Disorders of menstruation. Hoboken, NJ: Blackwell Publishing Ltd; 2011. [DOI:10.1002/9781444391824]

[3] Fernández-Martínez E, Onieva-Zafra MD, Parra-Fernández ML The impact of dysmenorrhea on quality of life among Spanish female university students. Int J Environ Res Public Health. 2019; 16(5):713. [DOI:10.3390/ijerph16050713] [PMID] [PMCID]

[4] Akhlaghi F, Zyrak N, Nazemian Sh. Effect of vitamin E on primary dysmenorrhea. Hayat. 2009; 15(1):13-9. [In Persian] http://hayat.tums.ac.ir/article-1-125-en.html

[5] Del Mar Fernández M, Regueira-Méndez C, Takkouche B. Psychological factors and premenstrual syndrome: A Spanish casecontrol study. PLoS One. 2019; 14(3):e0212557. [DOI:10.1371/ journal.pone.0212557] [PMID] [PMCID]

[6] Mohajerani M, Rezvani A. Comparison of the effect of herbal medicine Vitagnus (five fingers) with fluoxetine in controlling premenstrual syndrome in patients referred to the clinics of Islamic Azad University of Mashhad. J Med Sci. 2016; 6(2):1-8. [In Persian] http://medicine.sinaweb.net/article_528714.html

[7] UI Haq N, Gill S, Nasim A, Tahir M, Yasmin R, Batool F. Prevalence and impact of premenstrual syndrome among the female nursing students of Quetta. Asian J Nurs Educ Res. 2019; 9(2):239-42. [DOI:10.5958/2349-2996.2019.00050.8]

[8] Rebar R. Evaluation of amenorrhea, anovulation, and abnormal bleeding. In: Feingold KR, Anawalt B, Boyce A, Chrousos G, de Herder WW, Dungan $K$, et al, editors. Endotext. South Dartmouth, MA: MDText.com, Inc.; 2018. [PMID]

[9] Kalra P. Approach to Amenorrhea. In: Kalra P. Clinical Cases in Endocrinology. London: JP Medical Ltd; 2018. https://books. google.com/books?id=X3KSDwAAQBAJ\&dq

[10] Castelo-Branco C, Naumova I. Turner syndrome: Primary amenorrhea from adolescence to aging. In: Pérez-López F, ed. Postmenopausal Diseases and Disorders. Cham: Springer; 2019. p. 19-32. [DOI:10.1007/978-3-030-13936-0_2]

[11] Verrilli L, Blanchard H, Landry M, Stanic A. Prevalence and predictors of oligomenorrhea and amenorrhea in division 1 female athletes. Fertil Steril. 2018; 110(4):E245. [DOI:10.1016/j.fertnstert.2018.07.702]

[12] Huchon C, Fritel X. Epidemiology of menometrorrhagia. J Gynecol Obstet Biol Reprod (Paris). 2008; 37(8 Suppl 1):S307-16. [In French] [DOI:10.1016/S0368-2315(08)74770-7] [PMID]

[13] Philipp CS, Faiz A, Dowling N, Dilley A, Michaels LA, Ayers C, et al. Age and the prevalence of bleeding disorders in women with menorrhagia. Obstet Gynecol. 2005; 105(1):61-6. [DOI:10.1097/01.AOG.0000148889.15061.fb] [PMID]

[14] Dzhorbenadze MT, Kristesashvili D, Chopikashvili NA. The frequency and character of clinical manifestations of andro- genization among adolescent girls. Georgian Med News. 2005 (129):23-5. [In Russian] [PMID]

[15] Ahmadnia E, Maleki A, Moosavinasab N. Menstrual cycle pattern, its related disorders and associated factors in students of Zanjan, Iran. Qom Univ Med Sci J. 2014; 8(2):51-8. [In Persian] http://journal.muq.ac.ir/article-1-420-en.html

[16] Ravens-Sieberer U, Gosch A, Rajmil L, Erhart M, Bruil J, Duer W, et al. KIDSCREEN-52 quality-of-life measure for children and adolescents. Expert Rev Pharmacoecon Outcomes Res. 2005; 5(3):353-64. [DOI:10.1586/14737167.5.3.353] [PMID]

[17] Robitail S, Ravens-Sieberer U, Simeoni MC, Rajmil L, Bruil J, Power $\mathrm{M}$, et al. Testing the structural and cross-cultural validity of the KIDSCREEN-27 quality of life questionnaire. Qual Life Res. 2007; 16(8):1335-45. [DOI:10.1007/s11136-007-9241-1] [PMID]

[18] Nur Azurah AG, Sanci L, Moore E, Grover S. The quality of life of adolescents with menstrual problems. J Pediatr Adolesc Gynecol. 2013; 26(2):102-8. [DOI:10.1016/j.jpag.2012.11.004] [PMID]

[19] Soltani F, Artimani T. Evaluation the relationship between Menarch age $\&$ menstrual disorders. Avicenna J Nurs Midwifery Care. 2009;17(12):46-56. [In Persian] http://nmj.umsha.ac.ir/ article-1-1064-en.htm

[20] Sahin S, Ozdemir K, Unsal A. Evaluation of premenstrual syndrome and quality of life in university students. J Pak Med Assoc. 2014; 64(8):915-22. [PMID]

[21] Charu Sh, Amita R, Sujoy R, Thomas GA. 'Menstrual characteristics' and 'prevalence and effects of dysmenorrhea' on quality of life of medical students. Int J Collab Res Intern Med Public Health. 2012; 4(4):276-94. https://internalmedicine.imedpub. $\mathrm{com} /$ menstrual-characteristics-and-prevalence-and-effectofdysmenorrhea-on-quality-of-life-of-medical-students.pdf

[22] Unsal A, Ayranci U, Tozun M, Arslan G, Calik E. Prevalence of dysmenorrhea and its effect on quality of life among a group of female university students. Ups J Med Sci. 2010; 115(2):138-45 [DOI:10.3109/03009730903457218] [PMID] [PMCID]

[23] Delara M, Ghofranipour F, Azadfallah P, Tavafian SS, Kazemnejad A, Montazeri A. Health related quality of life among adolescents with premenstrual disorders: A cross sectional study. Health Qual Life Outcomes. 2012; 10:1. [DOI:10.1186/1477 7525-10-1] [PMID] [PMCID]

[24] Buddhabunyakan N, Kaewrudee S, Chongsomchai C, Soontrapa S, Somboonporn W, Sothornwit J. Premenstrual syndrome (PMS) among high school students. Int J Womens Health. 2017; 9:501-5. [DOI:10.2147/IJWH.S140679] [PMID] [PMCID]

[25] Victor FF, Souza Al, Barreiros CDT, de Barros JLN, da Silva FAC, Ferreira ALCG. Quality of life among university students with premenstrual syndrome. Rev Bras Ginecol Obstet. 2019; 41(5):312-7. [DOI:10.1055/s-0039-1688709] [PMID]

[26] Boustani F, Ziagham S, Jahangirimehr A, Honarmandpour A. Prevalence of premenstrual syndrome, premenstrual dysphoric disorder and associated factors among female high school students. J Inflamm Dis. 2019; 23(4):332-41. [In Persian-English] [DOI:10.32598/JQUMS.23.4.332] 